\title{
Comparison of Endoscopic Brush And Net Catheters As Sampling Tools To Analyze Lower Rectum Intestinal Mucus Samples of Patients With Ulcerative Colitis
}

\author{
Masanao Nakamura \\ Nagoya University Graduate School of Medicine \\ Keiko Maeda \\ Nagoya University Hospital \\ Kenta Yamamoto ( $\sim$ kenta-y@med.nagoya-u.ac.jp ) \\ Nagoya University Hospital \\ Takeshi Yamamura \\ Nagoya University Graduate School of Medicine \\ Tsunaki Sawada \\ Nagoya University Hospital \\ Yasuyuki Mizutani \\ Nagoya University Graduate School of Medicine \\ Eri Ishikawa \\ Nagoya University Graduate School of Medicine \\ Naomi Kakushima \\ Nagoya University Graduate School of Medicine \\ Kazuhiro Furukawa \\ Nagoya University Graduate School of Medicine \\ Tadashi lida \\ Nagoya University Graduate School of Medicine \\ Takuya Ishikawa \\ Nagoya University Graduate School of Medicine \\ Eizaburo Ohno \\ Nagoya University Graduate School of Medicine \\ Takashi Honda \\ Nagoya University Graduate School of Medicine \\ Hiroki Kawashima \\ Nagoya University Hospital \\ Masatoshi Ishigami \\ Nagoya University Graduate School of Medicine \\ Mitsuhiro Fujishiro \\ Nagoya University Graduate School of Medicine
}

\section{Research Article}

Keywords: microbiome, sampling, brush, ulcerative colitis, mass spectrometry

Posted Date: June 18th, 2021

DOI: https://doi.org/10.21203/rs.3.rs-618800/v1

License: (a) (i) This work is licensed under a Creative Commons Attribution 4.0 International License. Read Full License 


\section{Abstract}

Background and aims: The pathophysiology of ulcerative colitis remains unclear, but early lesions on the colorectal mucosal surface may play an important role in its etiology. Intestinal mucus samples, including inner and outer layers, are collected by net or brush catheters, but the quality of the samples obtained by each method has not been fully investigated. The aim of this study was to compare the microbiome and protein content of Intestinal mucus collected by net and brush catheters during colonoscopy.

Methods: Intestinal mucus samples from the lower rectum of four patients with ulcerative colitis were collected using a net catheter, a brush catheter, and intestinal fluid suction. Microbiome and protein content were analyzed using 16S rRNA gene sequencing and mass spectrometry.

Results: The patients demonstrated significant differences in microbiome alpha diversity $(p<0.05)$, but this difference was not observed between the sampling methods. Net catheter samples demonstrated higher total protein concentrations than brush catheter samples. Mucus-associated proteins (Mucin-2, Mucin-5B, Mucin-13, and IgGFc-binding protein) were more abundantly collected by nets in three patients with active ulcerative colitis, but more abundant by brushes in patients with inactive ulcerative colitis.

Bifidobacterium and some oral bacteria were similarly associated with ulcerative colitis activity.

Conclusions: Brush catheters are more likely to collect the intestinal mucus of inner layer, whereas net catheters are more likely to collect larger samples that include the outer mucus layer, as well as intestinal fluid. Two sampling methods with different types of work on the mucosa may lead different results among patients with mucosal vulnerabilities.

\section{Introduction}

The incidence of ulcerative colitis (UC) has continuously increased previously, but its pathophysiology has yet to be elucidated. Studies have proposed that the intestinal immune abnormalities in inflammatory bowel disease may be related to genetic and environmental factors [1, 2]. In particular, mental stress and overconsumption of fatty foods induce sympathetic hyperactivity, which reduces surface intestinal mucus. Reduced intestinal mucus results in increased antigen-presentation from diet and bacteria and a lower antiinflammatory response [3]. Decreased parasympathetic activity has also been shown to contribute to a collapse of the intestinal mucosal immune system [3]. The intestinal mucosal system, as well as the gut microbiome, is currently considered as the main mediator behind UC $[4,5]$.

Clinical studies have examined the gut microbiome through fecal samples [6, 7]. However, it is difficult to identify which part of the large intestine influences the microbiome data from fecal samples. Moreover, large inter-individual differences in gut microbiome data make it difficult to identify specific UC-causing organisms [8]. The gut microbiome can be examined by intestinal mucus sampling during gastrointestinal endoscopy [9], wherein a sample of intestinal mucus is extracted with a brush or net catheter. The brush catheter is used more frequently [10], but its collecting tip is small, which makes acquiring an adequate amount of samples more difficult. In contrast, the net catheter utilizes a loop that is approximately $2.5 \mathrm{~cm}$ in size. In clinical practice, it is used to retrieve resected colorectal polyps, but the intestinal mucus attached to the loop may be analyzed separately. Net catheters may be more useful for collecting larger amounts of intestinal mucus.

The mucus layer of the large intestine has inner and outer layers [11]. The inner layer is thicker and more resistant to bacteria, whereas the outer layer is less dense and more susceptible to bacterial colonization [12]. Brush sampling is more likely to provide information about the inner layer because it samples the mucosa perpendicular to the intestinal wall, whereas net sampling takes samples parallel to the intestinal wall.

The aim of this study was to collect samples of intestinal mucus obtained during colonoscopy and analyze these based on whether they were collected by a brush or net catheter. Microbiome and protein data were analyzed through 16S rRNA gene sequencing and mass spectrometry, respectively.

\section{Materials And Methods}

This was a single-center study that examined four patients with UC. We collected lower rectum intestinal mucus samples through colonoscopy. Three samples were taken from each patient (Fig. 1). Intestinal mucus from the anterior and right rectal walls were

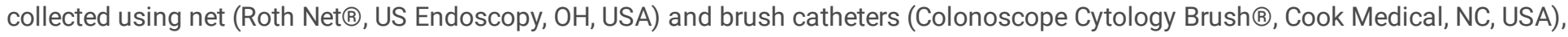


respectively, whereas intestinal fluid on the left rectal wall was collected by suction. Each catheter was divided in half, and each half was used for microbiome and protein analysis, respectively. Microbiome data was sequenced using MiSeq, and the results were compared with linear discriminant analysis effect size (LEfSe) taxonomic comparison [13]. Human proteins were identified and analyzed using mass spectrometry.

This study was approved by the Research Ethics Committee of the Nagoya University Hospital (protocol number 2015 - 0420, August $30,2016)$. Written informed consent was obtained from all patients prior to their enrollment in accordance with the Declaration of Helsinki. This study was registered in the University Hospital Medical Information Network Clinical Trials Registry (UMIN ID: 000020269). All clinical and stool sample information was anonymized before a database was constructed. The data of this research was based on the Nagoya gut microbiome database [14].

\section{Sample collection and 16S rRNA gene sequencing}

Gene sequencing was performed based on previously published methodology [15]. Samples were immediately stored at $-80^{\circ} \mathrm{C}$. Isolated DNA were amplified using universal primers (forward: 5'TCGTCGGCAGCGTCAGATGTGTATAAGAGACAGCCTACGGGNGGCWGCAG-3' and reverse: 5'GTCTCGTGGGCTCGGAGATGTGTATAAGAGACAGGACTACHVGGGTATCTAATCC-3') to target the V3-4 regions of the bacterial 16S rRNA. Sequencing data were obtained using the MiSeq Reagent Kit v3 (Illumina, San Diego, California, USA), with $2 \times 300$ reads and 600 cycles for microbial analysis. We followed the instruction tutorials and used QIIME2 [16] and DADA2; GreenGenes was used as a reference. The details are shown in the supplementary information 1.

\section{Mass spectrometry}

The samples were lysed using the Minute Total Protein Extraction Kit (Funakoshi, Tokyo, Japan). The supernatants were collected after centrifugation, and the protein concentrations of the supernatants were determined by bicinchoninic protein assays to determine the total amount of proteins in the samples. After reduction and alkylation, the proteins were digested with trypsin for $16 \mathrm{~h}$ at $37^{\circ} \mathrm{C}$. The peptides were analyzed by liquid chromatography mass spectrometry (LC MS) using an Orbitrap Fusionmass spectrometer (Thermo Fisher Scientific Inc., Waltham, Massachusetts, USA) coupled to an UltiMate3000 RSLC nano LC system (Dionex Co., Amsterdam, The Netherlands) using a nano high-performance LC capillary column, $150 \mathrm{~mm} \times 75 \mu \mathrm{m}$ i.d. (Nikkyo Technos Co., Japan) and a nanoelectrospray ion source. Reversed-phase chromatography was performed with a linear gradient (0 min, 5\% B; $100 \mathrm{~min}, 40 \% \mathrm{~B}$ ) with solvents A ( $2 \%$ acetonitrile with $0.1 \%$ formic acid) and B (95\% acetonitrile with $0.1 \%$ formic acid) at an estimated flow rate of 300 $\mathrm{nL} / \mathrm{min}$. A precursor scan was performed using a 400-1,600 mass-to-charge ratio $(\mathrm{m} / \mathrm{z})$ prior to MS/MS analysis. Tandem MS was performed by isolation at 0.8 Th with the quadrupole, high-energy collisional dissociation fragmentation at a normalized collision energy of $30 \%$, and rapid scan MS analysis was performed in the ion trap. Only precursors with charge states of 2-6 were sampled for MS2. The dynamic exclusion duration was set to $15 \mathrm{sec}$ with a $10 \mathrm{ppm}$ tolerance. The instrument was operated in the top speed mode with 3 sec cycles.

\section{Statistical analysis}

Categorical variables were compared using the chi-square or Fisher's exact test, whereas continuous variables were compared using the Mann-Whitney U test. Data were analyzed using IBM SPSS Statistics version 24 (SPSS Inc., Chicago, IL, USA).

Microbiome data were visualized and statistically analyzed using MicrobiomeAnalyst [17]. The alpha diversities of the observed species were calculated using the Shannon-Weiner and Simpson indices. The analysis was performed with the default settings, but the filtering and taxonomic levels were set as the feature level. Low and variance filters were not used because they take miner bacteria. Beta diversity was calculated using an analysis of similarities, a non-parametric statistical test, and the taxonomic level was selected as the feature and genus level. The alpha diversities of the bacteria at the phylum level were compared using the Kruskal-Wallis $\mathrm{H}$ test, whereas the alpha diversities of the bacteria at the genus level were compared using LEfSe in the default setting.

Protein data were processed using Proteome Discoverer 1.4 (Thermo Fisher Scientific) and identified with the MASCOT search engine version 2.6.0 (Matrix Science Inc., Boston, Massachusetts, USA). The peptides and proteins in the samples were identified against the human protein database in UniProt (release 2020_03), with precursor mass and fragment ion mass tolerances fixed at $10 \mathrm{ppm}$ and 0.8 $\mathrm{Da}$, respectively. Fixed modification was set with cysteine carbamidomethylation, and variable modifications were set for the oxidation of methionine. Two missed cleavages with trypsin were used. 


\section{Results \\ Patient data}

The clinical backgrounds of the four patients with UC are shown in Table 1 . We obtained 1,530,112 demultiplexed sequence counts (paired-end reads) with 948 features identified. The median sequencing depth was $86,207.5$, and the minimum frequency was 55,730 (Table 2). Both sequencing and feature data showed more variability when analyzed among patients than among the methods (supplementary information 2). However, all samples had sufficient sequence counts and quality for the analysis.

\section{Alpha diversity and relative bacterial abundance}

The alpha diversity of each patient is shown in the Table 2. The alpha diversity was significantly different among patients $(p<0.05)$, but there was no significant difference in the alpha diversities between the sampling methods (supplementary information 3 and 4 ). This difference can be clearly observed in the principal coordinates analysis plot because the samples formed clusters for each patient. This finding suggested that the microbiome data were more influenced by the patient than the method (supplementary information 5).

The percentages of phylum-level bacteria in each sample are shown in Fig. 2a. The microbiome was classified into nine phyla, and bacteroidetes, firmicutes, and proteobacteria were the most dominant. The major dominance differed among patients but not among methods. There was no significant difference in the phylum-level bacteria detected among the sampling methods (supplementary information 6).

The percentages of genus-level bacteria in each sample are shown in Fig. $2 \mathrm{~b}$. The relative abundance of each bacteria was different among patients and sampling methods. However, the heat map suggested that the differences were more significant among patients (Fig. 1c). The results from the LEfSe demonstrated significant bacterial differences among patients but not among methods (supplementary information 7).

\section{Bacterial data by sampling method}

Each sampling method demonstrated a similar microbiome for each patient, but the microbiome data showed variability among the patients. At the genus level, data from the brush and net catheter, brush catheter and intestinal fluid, and net catheter and intestinal fluid groups matched $85.9 \%, 80.75 \%$, and $84.93 \%$ of the time, respectively.

While the types of bacteria varied greatly among the patients, some bacteria consistently demonstrated a higher relative abundance when sampled with the net catheter compared to the brush catheter. Statistical comparison of each bacterium at the genus level is difficult because we only have four patients. Therefore, instead of comparing the mean of each bacteria, we searched for bacteria that showed the same trend in all patients. In four patients with widely varying relative abundance of bacteria, we searched for bacteria whose net sample was greater than the brush sampling in all patients. Five (Rothia, Granulicatella, Streptococcus, Butyricicoccus, and Bulleidia) genus were more abundant in the net catheter samples than the brush catheter samples (supplementary information 8 ). The relative abundance of these bacteria was higher in the intestinal fluid samples than in the net catheter samples. As such, intestinal fluid samples collected the most significant and representative amount of bacteria, followed by net catheters and brush catheters, respectively. This also suggested that net catheters may be collecting samples closer to the mucosal surface than brush catheters. In contrast no bacteria were detected in all patients with higher relative abundance in the brush than net.

\section{Mass spectrometry}

Specimens were collected from the anterior and right walls of the rectal mucosa using brush and net catheters, respectively. Patient characteristics are shown in Table 1. We identified the proteins in the net and brush catheter samples using mass spectrometry. Significantly higher protein concentrations were detected in the net catheter samples than the brush catheter samples (Fig. 3a). We used these sample concentrations to identify the total number of proteins in each patient. The medium number of identified proteins was 1183 (range 815-1537) for brush samples and 1104 (range 689-1423) for net samples Analysis of mucus samples collected from ex vivo colon biopsies by tandem mass spectrometry has identified 29 core proteins (Sjoerd et al). Consistent with this report, MUC2 mucin, the main constituent of intestinal mucus, and other mucins, such as MUC5 and MUC13A, IgGFc binding protein (FCGBP), were also detected and demonstrated similar concentrations regardless of the sampling method. 
Inflammatory protein markers (protein S100-A8, protein S100-A9) and a neurotrophic protein (myeloperoxidase) were also detected, but their expression levels were similar between the net and brush catheter samples (Fig. 3b). Some proteins were only identified in the net catheter samples; these are shown in Table 3.

\section{Relationship between microbiome and mass spectrometry for each patient}

We obtained samples from four patients. Of these, only patient No. 2 had a Clinical Activity Index (CAI) of 0, which means no active ulcerative colitis. The CAI was higher in patient No. 1, 4, 3, and 2, in that order. Mucin-2, Mucin-5B, Mucin-13 and IgGFc-binding protein (FCGBP) associated with intestinal mucosa of each patient are shown in Fig. 4a. Patients No.1.No.3.No.4 with active UC had higher abundance of these proteins collected in the net than in the brush. On the other hand, in the inactive UC patient No.2, more of these proteins were collected with the brush. Nine bacteria that showed a similar trend are shown in supplementary information 9 . This means that only patient 2 showed a different trend when comparing brushes and nets. The bacteria with the highest relative abundance are shown in Fig. 4b. A enough amount of Bifidobacterium was detected in patients No.1, No.3 and No.4, but the percentage detected in patient No.2 was very low. The genus Lachnospira, a butyrate-producing bacterium, was higher in the brushes of patient No. 2 than in the nets, and the other patients had the opposite. On the other hand, oral bacteria Staphylococcus and Dialister were collected in brushes of active UC patients No.1, No.3 and No.4 more than the net.

\section{Discussion}

Recent studies have demonstrated that the microbiome of patients with UC differ from healthy controls [18] because the mucosal inflammation in UC changes the mucosa-associated microbiota (MAM). Ulceration of the colonic epithelium further aggravates this condition, which perpetuates the cycle. Nishino et al. [19] reported that the relative abundance of Firmicutes and Proteobacteria in intestinal surface mucus was different among patients with UC and Crohn's disease, and normal controls. Fecal microbiota transplantation (FMT), probiotics, prebiotics, or synbiotics have been shown to be effective for UC [20, 21]; however, the pathogenesis of UC must be clarified before new therapeutic microbiome-based strategies are introduced.

The gut microbiome differs depending on the location in the large intestine. As such, we only collected samples from a single site, the lower rectum. Our endoscopic samples demonstrated a large amount of human DNA but little bacterial DNA [22]. Among the sampling techniques, brush catheter sampling has been reported to provide more bacterial DNA than biopsies, even if the amount of DNA obtained through brush catheter sampling is lower. We also expected that the brush catheter would acquire a smaller sample of proteins, which would make protein analysis difficult.

The colonic mucus layer has inner and outer layers. Both layers have the same protein profile; however, the inner mucus layer is denser and does not contain any bacteria or bacterial metabolites [11]. Therefore, the microbiome data is not expected to change, even if a larger proportion of the inner layer is sampled. In contrast, there is a difference in the microbiome of the mucus layer and intestinal fluid [23]. Each of our sampling techniques may have collected different proportions of mucus and intestinal fluid content, which would explain the difference in the microbiome data identified in our study. While both brushes scrape the mucus layer to collect a sample, the net catheter scrapes over a larger area, which may catch more intestinal fluid and mucus than the brush catheter.

Lavelle et al. also analyzed whether biopsy, brush, and laser capture microdissection affected the MAM data in five patients with UC and four controls [24]. Similar to our results, Lavelle et al. detected large inter-patient variabilities. While they were not able to identify definite UC-causing bacteria, their data did show higher relative abundance of Coriobacteriaceae, Bacteroidaceae, Ruminococcaceae [25], and Family XIII Incertae sedis in the mucosa of patients with US than in controls.

Our sample also showed a great variation among patients. Therefore, it was difficult to compare between patients in our study as well. However, comparison of brush and net proteins may suggest an association with activity. Abnormalities in mucus production have been reported in active UC. Abnormalities of the mucus system have been described in active UC. Reduction of MUC2 and FCGBP in the colonic mucus occurs prior to the onset of Reduction of MUC2 and FCGBP in the colonic mucus occurs prior to the onset of inflammation of UC and has been suggested to be related to the pathogenesis of UC. Mucins, component of mucus are classified into two different types: transmembrane mucins and gel-forming mucins. MUC2 and MUC5B are gel-forming MUC2 and MUC5B are gelforming secreted mucins and MUC13 is transmembrane mucin. Both types of mucins are secreted by the goblet cells. In samples from patients with active ulcerative colitis, more than twice as much protein associated with mucus was detected in the net. On the other hand, brush samples collected more protein associated with mucus in patient 2 , who had less active disease. This may indicate that 
the relatively weak horizontal force of the net may cause the mucus to peel off easier. In other words, these differences indicate mucosal weakness, which may be associated with higher symptom scores.

Nishino et al. [19] showed that Bifidobacterium levels were higher in UC patients than in healthy controls using brush samples. Lavelle A [24] also showed that UC patients had higher Bifidobacterium than controls and also showed higher abundance in Mucus than in Lumen. This is in agreement with our finding that only highly active patients had higher relative abundance of Bifidobacterium. The lower abundance of Lachnospira, a butyrate-producing bacterium collected by netting in less active UC patients than in brushes may indicate that the bacterium has settled in a deeper mucosal layer. On the other hand, in active UC patients, Staphylococcus Dialister, a resident oral bacterium, was collected more abundantly by brushes than nets, which may indicate that the bacterium resides in the deep mucosal layer.

Studies have proposed that patients with UC have different microbiomes compared to healthy controls, but whether this is the cause or result of the disease process remains unknown. Various bacteria have been also been identified as the possible triggers for UC, but these have yet to be proven. FMT has been suggested as an effective therapy for UC because it increases the production of short-chain fatty acids, particularly butyric acid [26]. Butyric acid decreases intestinal permeability and maintains the integrity of the intestinal epithelium, which reduces overall disease severity [27]. Our analysis was able to identify genus Butyricicoccus bacteria, a butyric acid bacterium, in our samples. Rothia and Streptococcus are commonly found in the oral cavity and are expected to be present near the mucosal lumen surface. Net catheter sampling has high potentials for future research because it can collect more bacteria.

Our study also demonstrated that net and brush catheter sampling collected the same types and amounts of mucosal proteins and inflammatory markers, except for leucine-rich alpha-2-glycoprotein (LRG) [28], which was detected in the brush catheter samples alone. As mentioned previously, the brush catheter collects samples perpendicular to the intestinal mucosa and is more likely to obtain portions of the inner mucus layer. This presumes that LRG may be more predominant in the inner layer rather than the mucosal surface.

This study has several limitations. First, our sample size was small. Second, we tried to perform a multi-omics analysis; however, there were significant differences in the microbiomes among the patients, which made the analysis impossible. Lastly, we did not analyze the proteins that were only detected in small amounts.

In conclusion, our study demonstrated the bacterial and protein content of intestinal mucosal samples taken with net and brush catheters among patients with UC. Brush catheters were more likely to acquire samples from the inner mucus layer, whereas net catheters were more likely to collect larger samples that include the outer mucus layer and intestinal fluid.

\section{Abbreviations}

UC: ulcerative colitis

LEfSe: linear discriminant analysis effect size

LC MS: liquid chromatography mass spectroscopy

MAM: mucosa-associated microbiome

FMT: fecal microbiome transplantation

\section{Declarations}

\section{- Ethics approval and consent to participate}

This study was approved by the Research Ethics Committee of the Nagoya University Hospital (protocol number 2015-0420, August 30, 2016). Written informed consent was obtained from all patients prior to their enrollment in accordance with the Declaration of Helsinki. This study was registered in the University Hospital Medical Information Network Clinical Trials Registry (UMIN ID: 000020269).

\section{- Consent for publication}

Not applicable 


\section{- Funding}

This work was partly supported by JSPS KAKENHI Grant Number 20 K07801.

\section{- Conflicts of interest}

This work was partly supported by JSPS KAKENHI Grant Number 20 K07801.

\section{- Funding}

This work was partly supported by JSPS KAKENHI Grant Number 20 K07801.

- Author contributions: Conception and design: Nakamura M, Maeda K, and Yamamoto K; analysis and interpretation of the data: Nakamura M, Yamamura T, Kakushima N, Maeda K, Yamamoto K, Ishikawa E, Ishikawa T, Mizutani Y, Ohno E, Sawada T, Furukawa K, and Honda T; drafting of the article: Nakamura M, Maeda K, and Yamamoto K; critical revision of the article for important intellectual content: lida T, Honda T, Ishigami M, and Kawashima H; and final approval of the article: Fujishiro M.

\section{- Acknowledgements}

We wish to thank Ms. Akina Ooishi of the Department of Gastroenterology and Hepatology of the Nagoya University Graduate School of Medicine for technical assistance in DNA isolation and 16S rRNA gene sequencing.

\section{- Availability of data and materials}

The datasets used and/or analyzed during the current study are available from the corresponding author on reasonable request.

\section{References}

1. Neurath MF. Targeting immune cell circuits and trafficking in inflammatory bowel disease. Nat Immunol 2019;20:970-9.

2. Kobayashi T, Siegmund B, Le Berre C, Wei SC, Ferrante M, Shen B, et al. Ulcerative colitis. Nat Rev Dis Primers 2020;6:74.

3. Teratani T, Mikami Y, Nakamoto N, Suzuki T, Harada Y, Okabayashi K, et al. The liver-brain-gut neural arc maintains the Treg cell niche in the gut. Nature 585;7826:591-6.

4. Jin L, Li L, Hu C, Paez-Cortez J, Bi Y, Macoritto M, et al. Integrative Analysis of Transcriptomic and Proteomic Profiling in Inflammatory Bowel Disease Colon Biopsies. Inflamm Bowel Dis. 2019;25:1906-18.

5. van der Post S, Jabbar KS, Birchenough G, Arike L, Akhtar N, Sjovall H, et al. Structural weakening of the colonic mucus barrier is an early event in ulcerative colitis pathogenesis. Gut 2019;68:2142-51.

6. Hattori S, Nakamura M, Yamamura T, Maeda K, Sawada T, Mizutani Y, et al. The microbiome can predict mucosal healing in small intestine in patients with Crohn's disease. J Gastroenterol 2020;55:1138-49.

7. Kummen M, Holm K, Anmarkrud JA, Nygård S, Vesterhus M, Høivik ML, et al. The gut microbial profile in patients with primary sclerosing cholangitis is distinct from patients with ulcerative colitis without biliary disease and healthy controls. Gut 2017;66:611-9.

8. Walker AW, Sanderson JD, Churcher C, Parkes GC, Hudspith BN, Rayment N, et al. High-throughput clone library analysis of the mucosa-associated microbiota reveals dysbiosis and differences between inflamed and non-inflamed regions of the intestine in inflammatory bowel disease. BMC Microbiol 2011;11:7.

9. Nagayama M, Yano T, Atarashi K, Tanoue T, Sekiya M, Kobayashi Y, et al. TH1 cell-inducing Escherichia coli strain identified from the small intestinal mucosa of patients with Crohn's disease. Gut Microbes. 2020;12:1788898.

10. Matsumoto H, Kuroki Y, Higashi S, Goda K, Fukushima S, Katsumoto R, et al. Analysis of the colonic mucosa associated microbiota (MAM) using brushing samples during colonic endoscopic procedures. J Clin Biochem Nutr 2019;65:132

11. Johansson MEV, Holmén Larsson JM, Hansson GC. The two mucus layers of colon are organized by the MUC2 mucin, whereas the outer layer is a legislator of host-microbial interactions. Proc Natl Acad Sci 2011;108(SUPPL. 1):4659-65.

12. Paone P, Cani PD. Mucus barrier, mucins and gut microbiota: The expected slimy partners? Gut. 2020 Dec;69(12):2232-2243.

13. Segata N, Izard J, Waldron L, et al. Metagenomic biomarker discovery and explanation. Genome Biol 2011;12:R60. 
14. Yamamoto $\mathrm{K}$, Ishigami M, Honda T, Takeyama T, Ito $\mathrm{T}$, Ishizu $\mathrm{Y}$, et al. Influence of proton pump inhibitors on microbiota in chronic liver disease patients. Hepatol Int 2019;13:234-44.

15. Zorron Cheng Tao Pu L, Yamamoto K, Honda T, Nakamura M, Yamamura T, Hattori S, et al. Microbiota profile is different for early and invasive colorectal cancer and is consistent throughout the colon. J Gastroenterol Hepatol 2020;35:433-7.

16. Bolyen E, Rideout JR, Dillon MR, Bokulich NA, Abnet CC, Al-Ghalith GA, et al. Reproducible, interactive, scalable and extensible microbiome data science using QIIME 2. Nat Biotechnol 2019;37:1091.

17. Chong J, Liu P, Zhou G, Xia J. Using Microbiome Analyst for comprehensive statistical, functional, and meta-analysis of microbiome data. Nat Protoc 2020;15:799-821.

18. Nemoto H, Kataoka K, Ishikawa H, Ikata K, Arimochi H, Iwasaki T, et al. Reduced diversity and imbalance of fecal microbiota in patients with ulcerative colitis. Dig Dis Sci. 2012;57:2955-64.

19. Nishino K, Nishida A, Inoue R, Kawada Y, Ohno M, Sakai S, et al. Analysis of endoscopic brush samples identified mucosaassociated dysbiosis in inflammatory bowel disease. J Gastroenterol 2018;53:95-106.

20. Dang $X, X u$ M, Liu D, Zhou D, Yang W. Assessing the efficacy and safety of fecal microbiota transplantation and probiotic VSL\#3 for active ulcerative colitis: A systematic review and meta-analysis. PLoS ONE 2020;15(3):e0228846.

21. Zhang X-F, Guan X-X, Tang Y-J, Sun JF, Wang XK, Wang WD, et al. Clinical effects and gut microbiota changes of using probiotics, prebiotics or synbiotics in inflammatory bowel disease: a systematic review and meta-analysis. Eur $\mathrm{J}$ Nutr in press.

22. Lavelle A, Lennon G, Docherty N, Balfe A, Mulcahy HE, Doherty G, et al. Depth-dependent differences in community structure of the human colonic microbiota in health. PLoS One 2013;8:78835.

23. Ouwerkerk JP, De Vos WM, Belzer C. Glycobiome: Bacteria and mucus at the epithelial interface. Vol. 27, Best Practice and Research: Clinical Gastroenterology. Bailliere Tindall Ltd; 2013. p. 25-38.

24. Lavelle A, Lennon G, O'Sullivan O, Docherty N, Balfe A, Maguire A, et al. Spatial variation of the colonic microbiota in patients with ulcerative colitis and control volunteers. Gut 2015;64:1553-61.

25. Willing BP, Dicksved J, Halfvarson J, Andersson AF, Lucio M, Zheng Z, et al. A pyrosequencing study in twins shows that gastrointestinal microbial profiles vary with inflammatory bowel disease phenotypes. Gastroenterology 2010;139:1844-1854.e1.

26. Fuentes S, Rossen NG, Van Der Spek MJ, Hartman JH, Huuskonen L, Korpela K, et al. Microbial shifts and signatures of long-term remission in ulcerative colitis after faecal microbiota transplantation. ISME J 2017;11:1877-89.

27. Shen ZH, Zhu CX, Quan YS, Yang ZY, Wu S, Luo WW, et al. Relationship between intestinal microbiota and ulcerative colitis: Mechanisms and clinical application of probiotics and fecal microbiota transplantation. World J Gastroenterol. 2018;24:5-14.

28. Shinzaki S, Matsuoka K, lijima H, Mizuno S, Serada S, Fujimoto M, et al. Leucine-rich Alpha-2 Glycoprotein is a Serum Biomarker of Mucosal Healing in Ulcerative Colitis. J Crohns Colitis 2017;11:84-91.

\section{Tables}

Table 1. Demographic and baseline characteristics of patients 


\begin{tabular}{|ll|}
\hline & $\mathrm{N}=4$ \\
\hline Gender, (female:male) & $3 / 1$ \\
\hline Age (median, range) & $46(22-70)$ \\
\hline Disease duration (month, range) & $900(24-144)$ \\
\hline Disease location, $N$ & \\
Extensive & 3 \\
Left-sided & 1 \\
Proctitis & 0 \\
\hline Treatment, $N$ & \\
\hline Oral 5-aminosalicycic acid, $N(\%)$ & $4(100)$ \\
Corticosteroids, $N(\%)$ & $1(25)$ \\
Biologic agents, $N(\%)$ & $1(25)$ \\
\hline Immunomodulators, $N(\%)$ & $1(25)$ \\
\hline Calcineurin inhibitors, $N(\%)$ & $0(0)$ \\
\hline Topical Agents, $N(\%)$ & $0(0)$ \\
\hline C-reactive protein (mg/dL) (median, range) & $0.12(0.01-0.39)$ \\
\hline Albumin (g/dL) (median, range) & $4.3(4.2-4.4)$ \\
\hline Rachmilewitz Clinical Activity Index (median, range) & $5(0-9)$ \\
\hline UCEIS score (median, range) & $3(2-4)$ \\
\hline
\end{tabular}

* UCEIS, Ulcerative Colitis Endoscopy Index of Severity 
Table 2

Demultiplexed sequence counts (paired-end reads) with 948 features identified by $16 \mathrm{~S}$ rRNA gene sequencing

\begin{tabular}{|c|c|c|c|c|c|c|c|c|c|c|}
\hline & & \multirow[b]{2}{*}{$\begin{array}{l}\text { Sequence } \\
\text { count }\end{array}$} & \multirow[b]{2}{*}{$\begin{array}{l}\text { Feature } \\
\text { Count }\end{array}$} & \multirow[b]{2}{*}{ Simpson } & \multirow[b]{2}{*}{ Chao1 } & \multirow[b]{2}{*}{ Shannon } & \multirow[b]{2}{*}{ Observed } & \multicolumn{3}{|c|}{ Match rate (genus level, \%) } \\
\hline & & & & & & & & $\begin{array}{l}\text { Brush } \\
\text { catheter }\end{array}$ & $\begin{array}{l}\text { Net } \\
\text { catheter }\end{array}$ & $\begin{array}{l}\text { Intestinal } \\
\text { fluid }\end{array}$ \\
\hline \multirow[t]{3}{*}{$\begin{array}{l}\text { Patient } \\
1\end{array}$} & $\begin{array}{l}\text { Brush } \\
\text { catheter }\end{array}$ & 137,043 & 98,629 & 0.8690262 & 158 & 2.812758 & 158 & - & 85.9 & 91.2 \\
\hline & $\begin{array}{l}\text { Net } \\
\text { catheter }\end{array}$ & 147,142 & 101,024 & 0.8807727 & 121 & 2.863048 & 121 & - & - & 85.3 \\
\hline & $\begin{array}{l}\text { Intestinal } \\
\text { fluid }\end{array}$ & 169,781 & 118,025 & 0.8765724 & 118 & 2.852898 & 118 & - & - & - \\
\hline \multirow[t]{3}{*}{$\begin{array}{l}\text { Patient } \\
2\end{array}$} & $\begin{array}{l}\text { Brush } \\
\text { catheter }\end{array}$ & 76,634 & 56,846 & 0.8840762 & 94 & 2.629995 & 94 & - & 80.6 & 71.9 \\
\hline & $\begin{array}{l}\text { Net } \\
\text { catheter }\end{array}$ & 116,490 & 85,041 & 0.9031833 & 108 & 2.83076 & 108 & - & - & 82.3 \\
\hline & $\begin{array}{l}\text { Intestinal } \\
\text { fluid }\end{array}$ & 79,657 & 57,825 & 0.8997583 & 103 & 2.857096 & 103 & - & - & - \\
\hline \multirow[t]{3}{*}{$\begin{array}{l}\text { Patient } \\
3\end{array}$} & $\begin{array}{l}\text { Brush } \\
\text { catheter }\end{array}$ & 146,611 & 105,408 & 0.9195404 & 172 & 3.386226 & 172 & - & 93.2 & 75.1 \\
\hline & $\begin{array}{l}\text { Net } \\
\text { catheter }\end{array}$ & 193,501 & 124,311 & 0.9407875 & 182 & 3.56386 & 182 & - & - & 78.5 \\
\hline & $\begin{array}{l}\text { Intestinal } \\
\text { fluid }\end{array}$ & 139,854 & 86,681 & 0.9549685 & 167 & 3.653543 & 167 & - & - & - \\
\hline \multirow[t]{3}{*}{$\begin{array}{l}\text { Patient } \\
4\end{array}$} & $\begin{array}{l}\text { Brush } \\
\text { catheter }\end{array}$ & 97,864 & 55,733 & 0.9432242 & 231 & 3.740973 & 231 & - & 83.9 & 84.8 \\
\hline & $\begin{array}{l}\text { Net } \\
\text { catheter }\end{array}$ & 136,630 & 85,734 & 0.9646357 & 271 & 4.085237 & 271 & - & - & 93.6 \\
\hline & $\begin{array}{l}\text { Intestinal } \\
\text { fluid }\end{array}$ & 88,905 & 55,730 & 0.9635789 & 262 & 4.074341 & 262 & - & - & - \\
\hline \multirow[t]{3}{*}{ Average } & $\begin{array}{l}\text { Brush } \\
\text { catheter }\end{array}$ & 114,538 & 79,154 & 1 & 164 & 3 & 164 & - & 85.9 & 80.75 \\
\hline & $\begin{array}{l}\text { Net } \\
\text { catheter }\end{array}$ & 148,441 & 99,028 & 1 & 171 & 3 & 171 & - & - & 84.925 \\
\hline & $\begin{array}{l}\text { Intestinal } \\
\text { fluid }\end{array}$ & 119,549 & 79,565 & 1 & 163 & 3 & 163 & - & - & - \\
\hline
\end{tabular}


Table 3

Proteins detected in net catheter samples only

\begin{tabular}{|c|c|c|c|}
\hline Accession & Protein name & MW & Score \\
\hline P8217 & Chymotrypsin-like elastase family member $2 \mathrm{~A}$ & 85.4 & $8,965.87$ \\
\hline P09923 & Intestinal-type alkaline phosphatase & 72.5 & $2,977.77$ \\
\hline P04118 & Colipase & 35.4 & $2,679.83$ \\
\hline P08861 & Chymotrypsin-like elastase family member 3B & 141.5 & $2,611.26$ \\
\hline P13688 & Carcinoembryonic antigen-related cell adhesion molecule 1 & 28.9 & $1,804.4$ \\
\hline P22748 & Carbonic anhydrase 4 & 57.5 & $1,700.48$ \\
\hline P04054 & Phospholipase A2 & 15.3 & $1,693.51$ \\
\hline Q9BYE9 & Cadherin-related family member 2 & 25.6 & $1,667.72$ \\
\hline P05107 & Integrin beta2 & 28.9 & $1,371.29$ \\
\hline P35580 & Myosin-10 & 29.2 & $1,252.92$ \\
\hline P13797 & Plastin-3 & 191.5 & $1,023.11$ \\
\hline A0A075B6K5 & Immunoglobulin lambda variable 3-9 & 11.9 & 869.48 \\
\hline P49913 & Cathelicidin antimiicrobial peptide & 60.1 & 851.99 \\
\hline P14136 & Glial fibrillary acidic protein & 63.1 & 840.2 \\
\hline 043451 & Malate glucoamylase, intestinal & 36.2 & 804.48 \\
\hline Q00610 & Clathrin heavy chains 1 & 50.1 & 784.71 \\
\hline P08575 & Receptor-type tyrosine-protein phosphatase C & 39.1 & 644.21 \\
\hline P14923 & Junction plakoglobin & 16.1 & 536.23 \\
\hline A0A075B6S6 & Immunoglobulin kappa variable 2D-30 & 49.8 & 535.85 \\
\hline Q09666 & Neuroblast differentiation-associated protein AHNAK & 78.8 & 474.64 \\
\hline P51970 & NADH dehydrogenase (ubiquinone) 1 alpha subcomplex subunit 8 & 12.7 & 372.85 \\
\hline 075830 & Serpin12 & 13.5 & 358.93 \\
\hline P62834 & Ras-related protein Rap-1A & 13.2 & 357.57 \\
\hline P08962 & CD63 antigen & 12.6 & 320.95 \\
\hline P29350 & Tyrosine-protein phosphatase non-receptor type 6 & 12.5 & 305.96 \\
\hline Q06210 & Glutamine-fructose-6-phosphate aminotransferase (isomerizing) 1 & 12.6 & 301.47 \\
\hline Q6UX82 & Ly6/PLAUR domain-containing protein 8 & 12.3 & 293.9 \\
\hline P19075 & Tetraspanin-8 & 12.4 & 290.21 \\
\hline P26641 & Elongation factor 1-gamma 1 & 84.7 & 290.03 \\
\hline Q06323 & Proteasome activator complex subunit 1 & 56.8 & 278.75 \\
\hline Q9UFN0 & Protein NipSnap homolog $3 \mathrm{~A}$ & 81.7 & 262.03 \\
\hline P19338 & Nucleolin & 38.9 & 259.06 \\
\hline 075131 & Copine-3 & 25.2 & 252.41 \\
\hline 000584 & Ribonuclease T2 & 209.7 & 219.07 \\
\hline
\end{tabular}




\begin{tabular}{|c|c|c|c|}
\hline Accession & Protein name & MW & Score \\
\hline Q8WZ42 & Titin & 46.6 & 206.99 \\
\hline Q99798 & Aconitate hydratase, mitochondrial & 83.6 & 197.84 \\
\hline P27216 & Annexin A13 & 228.9 & 193.28 \\
\hline Q6UXH1 & Cystein-rich with EGF-like domain protein 2 & 20.1 & 160.39 \\
\hline P30048 & Thioredoxin-dependent peroxide reductase, mitochondrial & 628.7 & 153.36 \\
\hline 075477 & Erlin-1 & 76.6 & 147.2 \\
\hline P11310 & Medium-chain specific acyi-CoA dehydrogenase, mitochondrial & 48.5 & 146.29 \\
\hline A0A087WSY6 & Immunoglobulin kappa variable 3D-15 & 16.3 & 138.52 \\
\hline P61457 & Pterin-4-alpha-carbinolamine dehydratase & 70.8 & 125.63 \\
\hline Q9H4A4 & Aminopeptidase B & 28.7 & 124.13 \\
\hline Q5TZA2 & Rootletin & 28.4 & 123.3 \\
\hline Q5T0J7 & Testis-expressed sequence 35 protein & 12 & 118.9 \\
\hline P01709 & Immunoglobulin lambda variable $2-8$ & 21 & 116.05 \\
\hline P01706 & Immunoglobulin lambda variable $2-11$ & 147.4 & 115.43 \\
\hline Q16881 & Thioredoxin reductase 1 & 29.5 & 106.31 \\
\hline AOAOAOMRZ8 & Immunoglobulin kappa variable 3D-11 & 228.4 & 105.43 \\
\hline A0A0B4J1U7 & Immunoglobulin heavy variable 6 - 1 & 46.1 & 91.95 \\
\hline P01742 & Immunoglobulin heavy variable 1-69 & 26.5 & 87.15 \\
\hline Q86YQ8 & Copine-8 & 26 & 84.42 \\
\hline Q15645 & Pachtene checkpoint protein 2 homolog & 70.9 & 81.42 \\
\hline Q16891 & MICOS complex subunit MIC60 & 56.5 & 56.71 \\
\hline Q05315 & Galectin-10 & $3,813.7$ & 56.69 \\
\hline Q86TE4 & Leucine zipper protein 2 & 67.5 & 47.66 \\
\hline
\end{tabular}

\section{Figures}


anterior wall

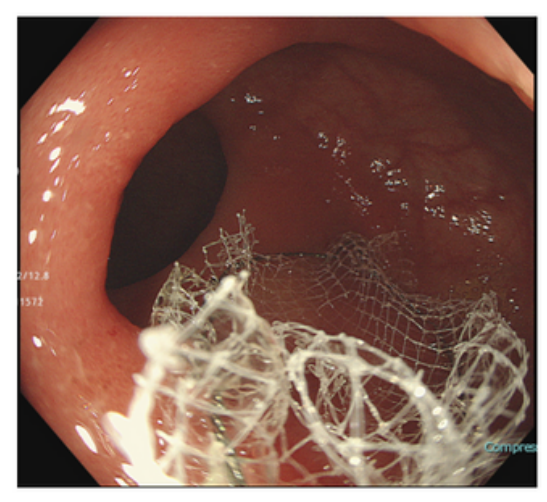

Net catheter right side wall

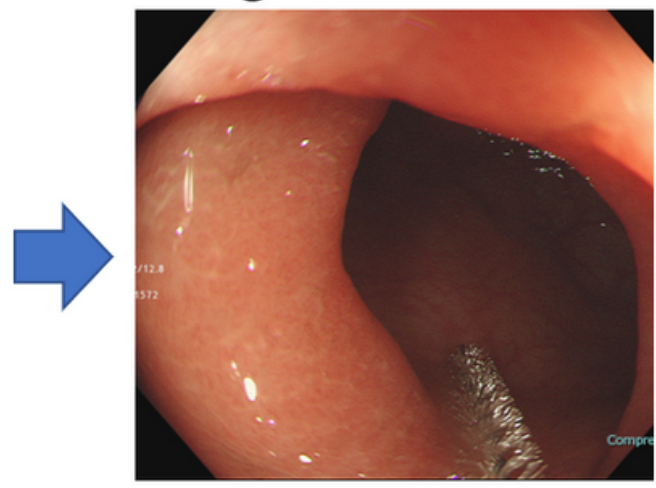

Brush catheter

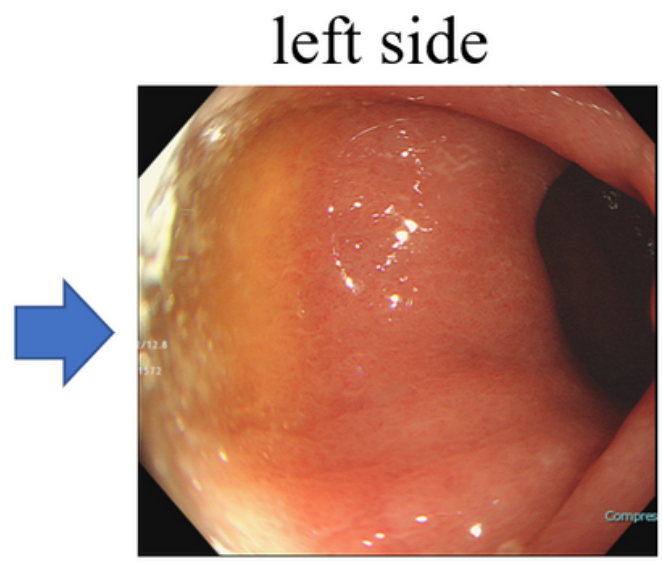

Fluid suction

\section{Figure 1}

Endoscopic images of the lower rectum. This photograph shows the mucus in the lower rectum being collected by a net catheter, brush catheter, and intestinal suction.

a

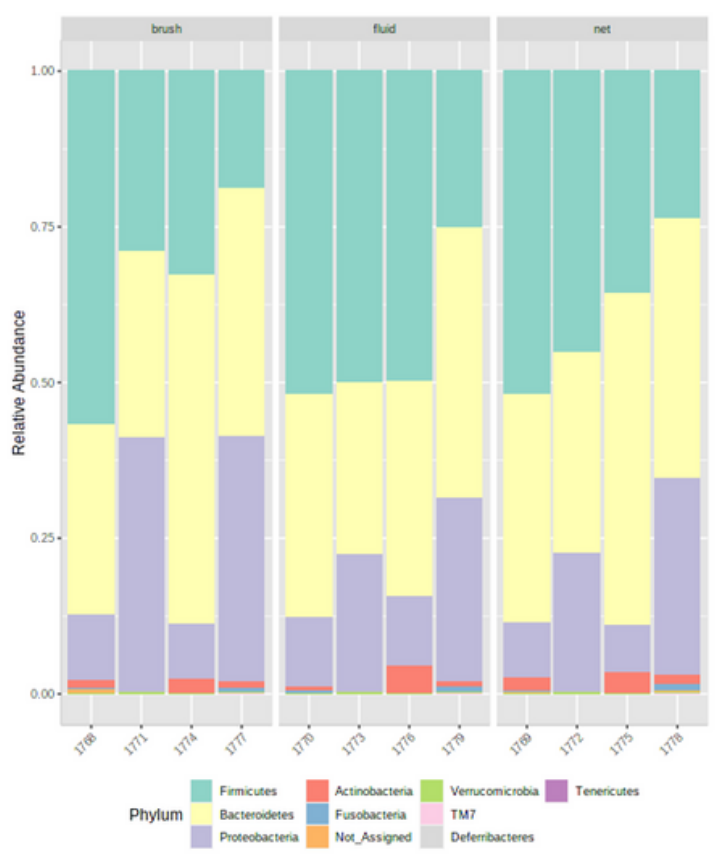

b

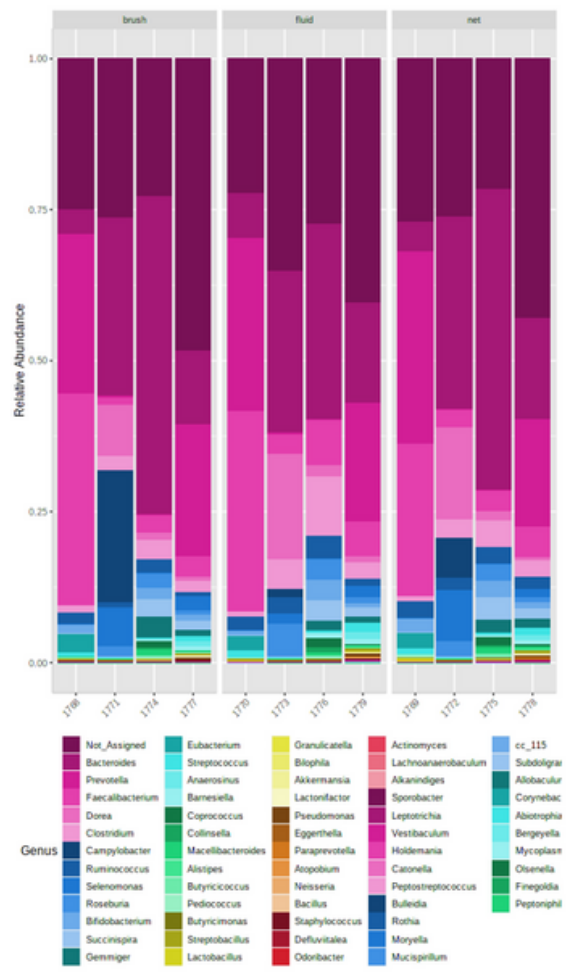

C

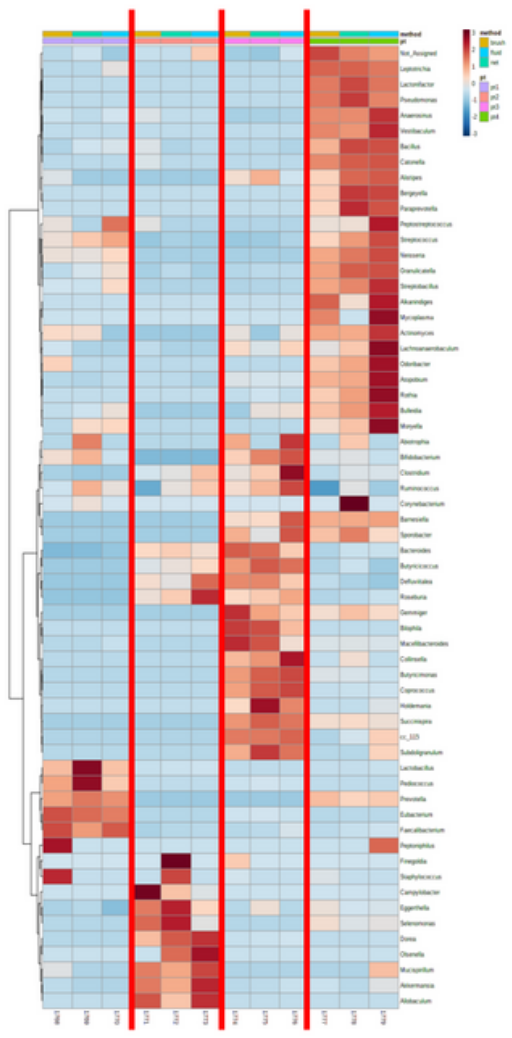

Figure 2

16S rRNA gene sequencing a. This image shows the proportions of the microbiome at the phylum level. $\mathrm{b}$. This image shows the proportions of the microbiome at the genus level. c. This image shows the heatmap of the microbiome at the genus level. 


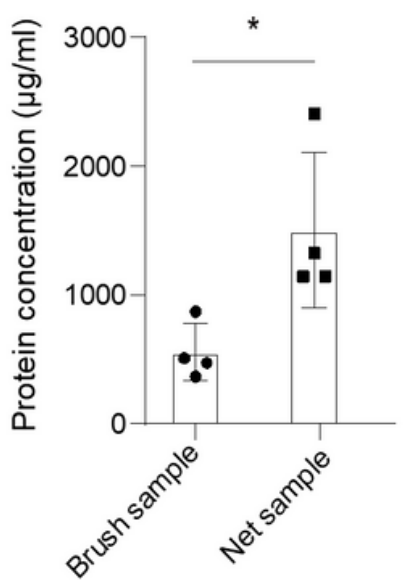

(a) (b)
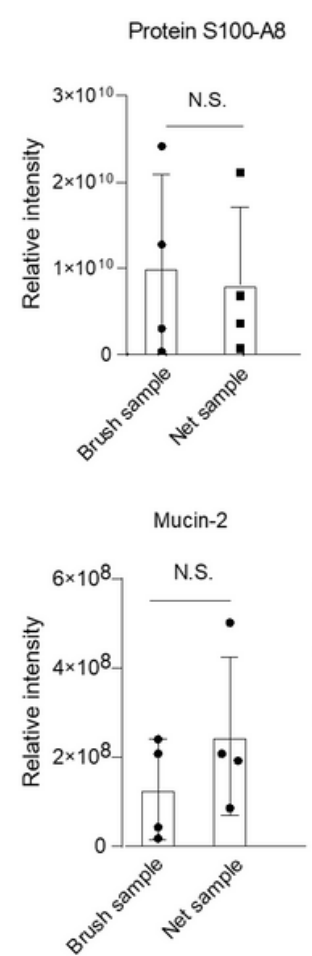

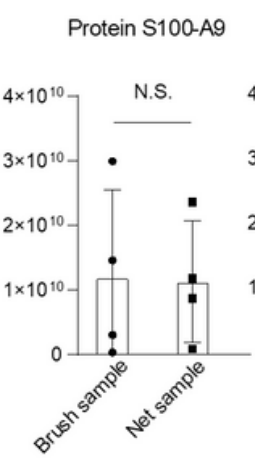

Myeloperoxitase Leucine-rich alpha 2 glycoprotei (LRG)
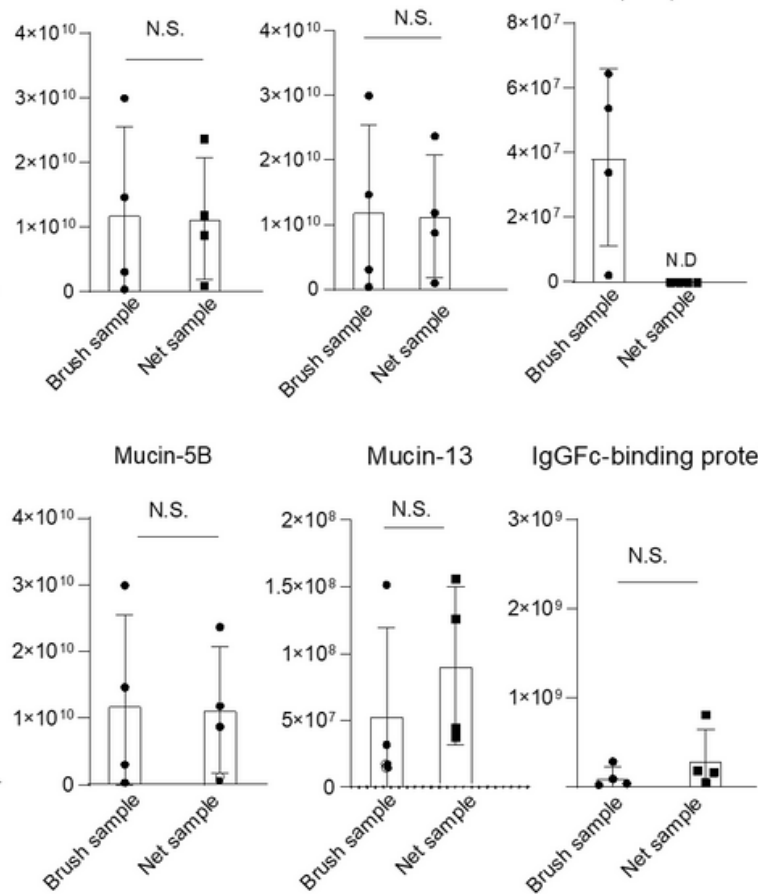

IgGFc-binding protein

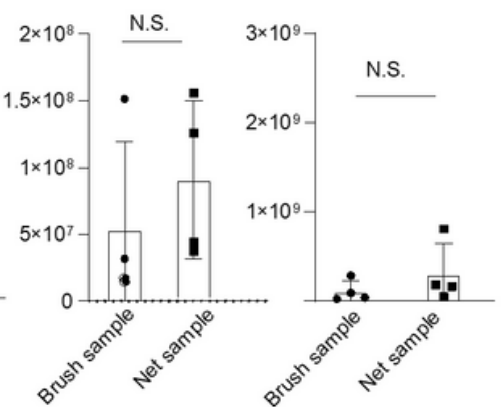

\section{Figure 3}

a: Protein concentrations in the net and brush catheter samples b: Proteins collected by the net and brush catheter samples
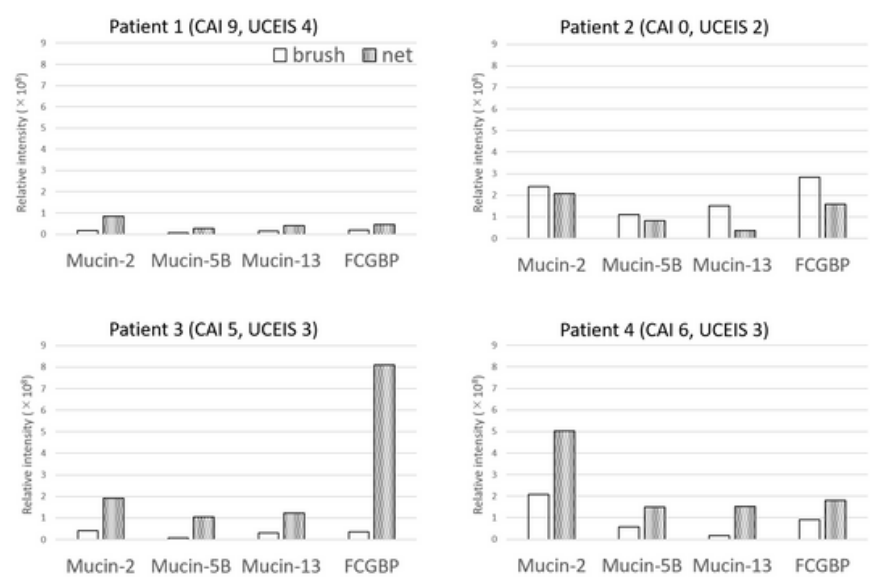

(a)
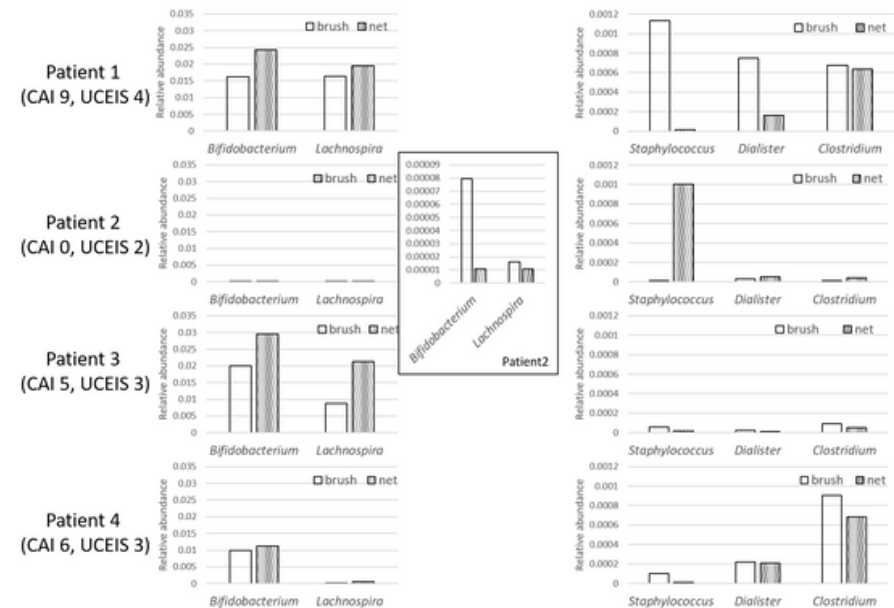

(b)

\section{Figure 4}

a: The amount of protein associated with the mucus layer obtained by brush or net is shown for each patient. Only patient 2 is a patient with no activity (CAI:0). These proteins were more collected in the brushes in patient 2 versus the nets in the other patients. In addition, the net collected twice as much as the brush. Because the vertical force on the mucus layer is weaker with the net than with the brush, the mucus layer may peel off more easily in active patients. b: Bifidobacterium in patient 2 is significantly less. In addition, more bacteria were collected on the brush than on the net. On the other hand, the oral bacteria Staphylococcus, Dialister was collected more on the brush than on the net in three patients. If brushes are collected from a deeper mucus layer than nets, this may indicate that there is less Bifidobacterium and more oral bacteria in the deeper mucus layer of active patients. CAl: Clinical activity index, UCEIS: Ulcerative Colitis Endoscopic Index of Severity B: Proteins collected by the net and brush catheter samples

\section{Supplementary Files}


This is a list of supplementary files associated with this preprint. Click to download.

- supplementaryinformationall.pdf 